\title{
Mobile Healthcare Information Management utilizing Cloud Computing and Android OS
}

\author{
Jyoti Lokhande ${ }^{1}$, Prof. Rahul Gaikwad ${ }^{2}$ \\ ${ }^{I} P G$ Student, Godavari College of Engineering, Jalgaon, Maharashtra, India \\ ${ }^{2}$ Assistant Professor, Godavari College of Engineering, Jalgaon, Maharashtra, India
}

Received on: 16 July, 2021

Revised on: 11 August, 2021

Published on: 13 August, 2021

\begin{abstract}
Anywhere anytime availability of $e$ health applications to everyone. Android $O S$ is open source platform available to develop the different applications. Using cloud computing functionalities e health application will be used to ease everyday life.
\end{abstract}

Keywords- Android, Cloud computing, MADIP system, ubiquitous access

\section{I - INTRODUCTION}

M obile healthcare systems focus towards achieving two specific goals: the availability of e-health applications and medical information anywhere and anytime and the invisibility of computing. Mobile pervasive healthcare technologies can support a wide range of applications and services including mobile telemedicine, patient monitoring, location-based medical services, emergency response and management, personalized monitoring and pervasive access to healthcare information, providing great benefits to both patients and medical personnel. The realization however of health information management through mobile devices introduces several challenges, like data storage and management (e.g., physical storage issues, availability and maintenance), interoperability and availability of heterogeneous resources, security and privacy (e.g., permission control, data anonymity, etc.), unified and ubiquitous access. One potential solution for addressing all aforementioned issues is the introduction of Cloud
Computing concept in electronic healthcare systems. Cloud Computing provides the facility to access shared resources and common infrastructure in a ubiquitous and pervasive manner, offering services on demand over the network to perform operations that meet changing needs in electronic healthcare application. In this context @ Health Cloud has been developed; a pervasive healthcare information management system for mobile devices utilizing Cloud Computing and Android Operating System (OS).

\section{II -MOBILE HEALTHCARE SYSTEMS AND CLOUD COMPUTING}

Several studies have demonstrated that the limited access to patient-related information during decision-making and the ineffective communication among patient care team members are proximal causes of medical errors in healthcare. Thus, the pervasive and ubiquitous access to healthcare data is considered essential for the proper diagnosis and treatment procedure. Cloud Computing is a model for enabling convenient, on-demand network access to a shared group of configurable computing resources (e.g., networks, servers, storage, applications, and services) that can be rapidly provisioned and released with minimal management effort or service provider interaction.

\section{III -RELATED WORK}

The main purpose of the work is to provide seamless and consistent communication flow between home health care 


\section{International Journal of Innovations in Engineering and Science, www.ijies.net}

and primary care providers using devices like PDAs and Tablet PCs. Smart cards and web interfaces have been used for storing patient records electronically. The MADIP system is a distributed information platform allowing widearea health information exchange based on mobile agents. The majority of the aforementioned works is based on proprietary architectures and communication schemes and requires the deployment of specific software components. Furthermore, the works focus mostly on delivering data to healthcare applications and do not address issues of data management and interoperability issues introduced by the heterogeneous data resources found in modern healthcare systems. The usage of Cloud Computing provides data management and access functionality overcoming the aforementioned issues as discussed in previous sections. The concept of utilizing Cloud Computing in the context of healthcare information management is relatively new but is considered to have great potential.

\section{IV- APPLICATION OVERVIEW}

This section discusses the main features of the @ Health Cloud application and presents implementation details. The prevalent functionality of the application is to provide medical experts and patients with a mobile user interface for managing healthcare information. The latter interprets into storing, querying and retrieving medical images, patient health records and patient-related medical data (e.g., bio signals). The data may reside at a distributed Cloud Storage facility, initially uploaded/stored by medical personnel through a Hospital Information System (HIS). In order to be interoperable with a variety of Cloud Computing infrastructures, the communication and data exchange has to be performed through non-proprietary, open and interoperable communication standards. @ HealthCloud utilizing Web Services connectivity and Android OS supports the following functionality: Seamless connection to Cloud Computing storage: The main application allows users to retrieve, modify and upload medical content (medical images, patient health records and biosignals) utilizing Web Services and the REST API. The content resides remotely into the distributed storage elements but access is presented to the user as the resources are located locally in the device.

\section{V - PROPOSED SYSTEM ARCHITECTURE AND IMPLEMENTATION DETAILS}

The main components of a Cloud Computing Service usually are the platform frontend interface that communicates directly with users and allows the management of the storage content. The interface can be a web client or a standalone application. The Cloud Storage Facilities manages the physical infrastructure (e.g., storage elements) and is also responsible for performing maintaining operations (e.g., backing up data, etc.)

The Cloud Platform interface is also connected to the Cloud Service module, which handles and queues user requests. Finally, the Cloud Infrastructure module manages user account, accessibility and billing issues. Android is a mobile operating system running on the Linux kernel. Several mobile device vendors already support it. The platform is adaptable to larger and traditional smart phone layouts and supports a variety of connectivity technologies (CDMA, EV-DO, UMTS, Bluetooth, and Wi-Fi). It supports a great variety of audio, video and still image format, making it suitable for displaying medical content. Finally, it supports native multi-touch technology, which allows better manipulation of medical images and generally increases the application's usability. The Cloud Service client running on Android OS consists of several modules. The Patient Health Record application acquires and displays patient records stored into the cloud. The Medical Imaging module is responsible for displaying medical images on the device.

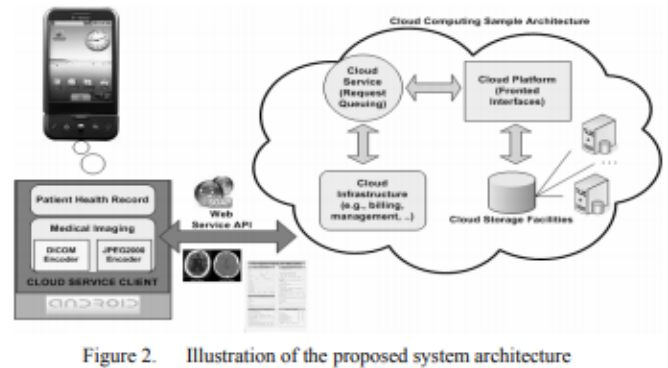

It decodes images in DICOM format displaying both image and heard information data. The communication with the Cloud is performed through an implementation of Web Services REST API that is supported natively by Android. The inherent interoperability that comes with using vendor, platform, and language independent XML technologies and the ubiquitous HTTP as a transport mean that any application can communicate with any other application using Web services. Data in Cloud are seamlessly stored and presented to the user as if they reside locally. This means that the Cloud repository is presented as a virtual folder and does not provide the features of a database scheme. In order to provide the user with data querying functionality, medical records and related data (images and biosignals) are stored into a SQLite file. SQLite is the database platform supported by Android. The file resides into a specific location at the Cloud and is retrieved on the 


\section{International Journal of Innovations in Engineering and Science, www.ijies.net}

device every time user needs to query data. The query is performed locally and the actual location of the data in the cloud is revealed to the applications. The database file is updated and uploaded into the Cloud every time user modifies data.

\section{VI -INITIAL EVALUATION FROM THE SYSTEM IN PRACTICE}

In order to prove the system's usability, some initial experiments evaluating the system's performance have been conducted. Experiments concern the time needed to transmit data to the Amazon S3 Cloud storage service. Due to the fact that textual data like a patient's health record or a bio signal sequence do not consist of large data files and do not require high bandwidth, the presented results involve the transmission of medical images. The @HealthCloud application as presented in previous sections has been used on a HTC G1 mobile phone running Android OS version 1.6. A number of medical images of different modalities (MR, CT, PET, OT and Ultrasound) and different file sizes have been sed.

The concept of Cloud Computing and applications similar to the one presented in this article will attract the interest of scientists, developers and industrial partners working in the field of biomedical informatics. This paper has presented @HealthCloud: a prototype implementation of a mobile healthcare information management system based on Cloud Computing and Android OS. The system enables the management of patient health records and medical images (supporting DICOM format and JPEG2000 coding) and utilizes the Amazon's S3 Cloud Storage Service. Future work might include improving security by implementing advanced user authentication techniques on the mobile device (e.g. through voice recognition) and deploying the platform in real healthcare environment for evaluating the system in terms of user acceptability and performance.

\section{REFERENCES}

[1] Upkar Varshney, "Pervasive Healthcare", IEEE Computer Magazine vol. 36, no. 12, 2003, pp. 138-140.

[2] The Android mobile OS by GoogleTM, http://www.android.com/

[3] Sabine Koch, Maria Hägglund, Isabella Scandurra, Dennis Moström, "Towards a virtual health record for mobile home care of elderly citizens", presented in MEDINFO 2004, Amsterdam, 2004.

[4] Alvin T.S. Chan, "WWW_smart card: towards a mobile health care management system", International Journal of Medical Informatics vol. 57, 2000, pp. 127-137.

[5] Chuan Jun Su, "Mobile multi-agent based, distributed information platform (MADIP) for wide-area e-health monitoring", Computers in Industry, vol. 59, 2008, pp. 55-68.

[6] Khawar Hameed, "The application of mobile computing and technology to health care services", Telematics and Informatics, vol. 20, 2003, pp. 99-106.

[7] Eneida A. Mendonça, Elizabeth S. Chen, Peter D. Stetson, Lawrence K. McKnight, Jianbo Lei, James J. Cimino, "Approach to mobile information and communication for health care", International Journal of Medical Informatics, vol 73, 2004, pp. 631-638.

[8] L.L. Leape, "Error in medicine”, J. Am. Med. Assoc, vol. 272, 1994, pp. 1851-1857.

[9] J.T. Reason, Human Error, Cambridge University Press, Cambridge, 1990.

[10] Maglogiannis I., Doukas C., Kormentzas G., Pliakas T., "WaveletBased Compression With ROI Coding Support for Mobile Access to DICOM Images Over Heterogeneous Radio Networks", IEEE Transactions on Information Technology in Biomedicine, vol.13, no.4, pp.458-466, July 2009.

[11] George Reese, Cloud Application Architectures: Building Applications and Infrastructure in the Cloud, O'Reilly Media, Paperback (April 17, 2009), ISBN: 0596156367.

[12] GoGrid Storage Services, http://www.gogrid.com

[13] iCloud, http://www.icloud

[14] Amazon Web Services (AWS), http://aws.amazon.com/

[15] DropBox, https://www.dropbox.com 